\title{
Potential Role of Aspirin in the Prevention of Aneurysmal Subarachnoid Hemorrhage
}

\author{
Robert M. Starke ${ }^{a}$ Nohra Chalouhi $^{b}$ Dale Ding ${ }^{a}$ David M. Hasan ${ }^{c}$ \\ ${ }^{a}$ Department of Neurological Surgery, University of Virginia, Charlottesville, Va., ${ }^{b}$ Department of Neurological \\ Surgery, Thomas Jefferson University, Philadelphia, Pa., and ' ${ }^{\mathrm{D}}$ Department of Neurological Surgery, University of lowa, \\ Cedar Rapids, lowa, USA
}

\author{
Key Words \\ Aneurysm · Cerebral · Inflammation · Rupture . \\ Subarachnoid hemorrhage · Aspirin · Antithrombotic . \\ Cyclooxygenase
}

\begin{abstract}
Background: Inflammation is a key element behind the pathophysiology of cerebral aneurysm formation and rupture. Aspirin is a potent inhibitor of cyclooxygenase-2 (COX), which plays a critical role in the expression of immune modulators known to contribute to cerebral aneurysm formation and rupture. Currently, there are no pharmacological therapies for patients with cerebral aneurysms. Both endovascular and microsurgical interventions may be associated with significant morbidity and mortality. Potentially, a medical alternative that prevents aneurysm progression and rupture may be a beneficial therapy for a significant number of patients. Summary: In animal models, treatment with aspirin and genetic inactivation of COX-2 decreases aneurysm formation and rupture. Selective inhibition of COX-1 did not decrease aneurysm rupture, suggesting that selection inhibition of COX-2 may be critical in thwarting aneurysm progression. Walls of ruptured human intracranial aneurysms have higher levels of COX-2 and microsomal prostaglandin E2 synthase 1 (mPGES-1), both of which are known to be inhibited by aspirin. In a pilot study, patients undergoing microsurgical clipping had attenuated expression of COX-2, mPGES-1, and macrophages in aneurysm walls after 3 months of aspirin therapy versus those that did not receive aspirin. Addition-
\end{abstract}

ally, in patients undergoing endovascular therapy, local circulating expression of chemokines and COX-2 were increased in blood samples taken from within aneurysm domes as compared to peripheral blood sample controls. Treatment with aspirin also resulted in decreased expression of COX-2 within leukocytes within aneurysms as compared to peripheral blood samples. Novel molecular imaging with ferumoxytol-enhanced MRI may help in the identification of patients at increased risk for aneurysm rupture and assessment of a response to aspirin therapy. Key Messages: Aspirin has been found to be a safe in patients harboring cerebral aneurysms and clinical studies provide evidence that it may decrease the overall rate of rupture. Furthermore, aspirin is an accessible and inexpensive medicine for patients who may not have access to endovascular or microsurgical treatment or for patients who are deemed low risk of aneurysm rupture, high risk for intervention, or both. Future clinical trials are indicated to determine the overall effect of aspirin on aneurysm progression and rupture. This review provides an update on the potential mechanisms and benefits of aspirin in the treatment of cerebral aneurysms.

๑ 2015 S. Karger AG, Basel

\section{Introduction}

Subarachnoid hemorrhage (SAH) constitutes 5-10\% of strokes in the United States [1]. Intracranial aneurysm rupture is the etiology of approximately $85 \%$ of spontaneous SAH [2]. While treatment of intracranial aneurysms

\section{KARGER 125}

(c) 2015 S. Karger AG, Base

$1015-9770 / 15 / 0396-0332 \$ 39.50 / 0$

E-Mail karger@karger.com

www.karger.com/ced
David M. Hasan, MD

Department of Neurological Surgery

University of Iowa Hospitals and Clinics, 200 Hawkins Dr.

Iowa City, IA 52242 (USA)

E-Mail david-hasan@uiowa.edu 


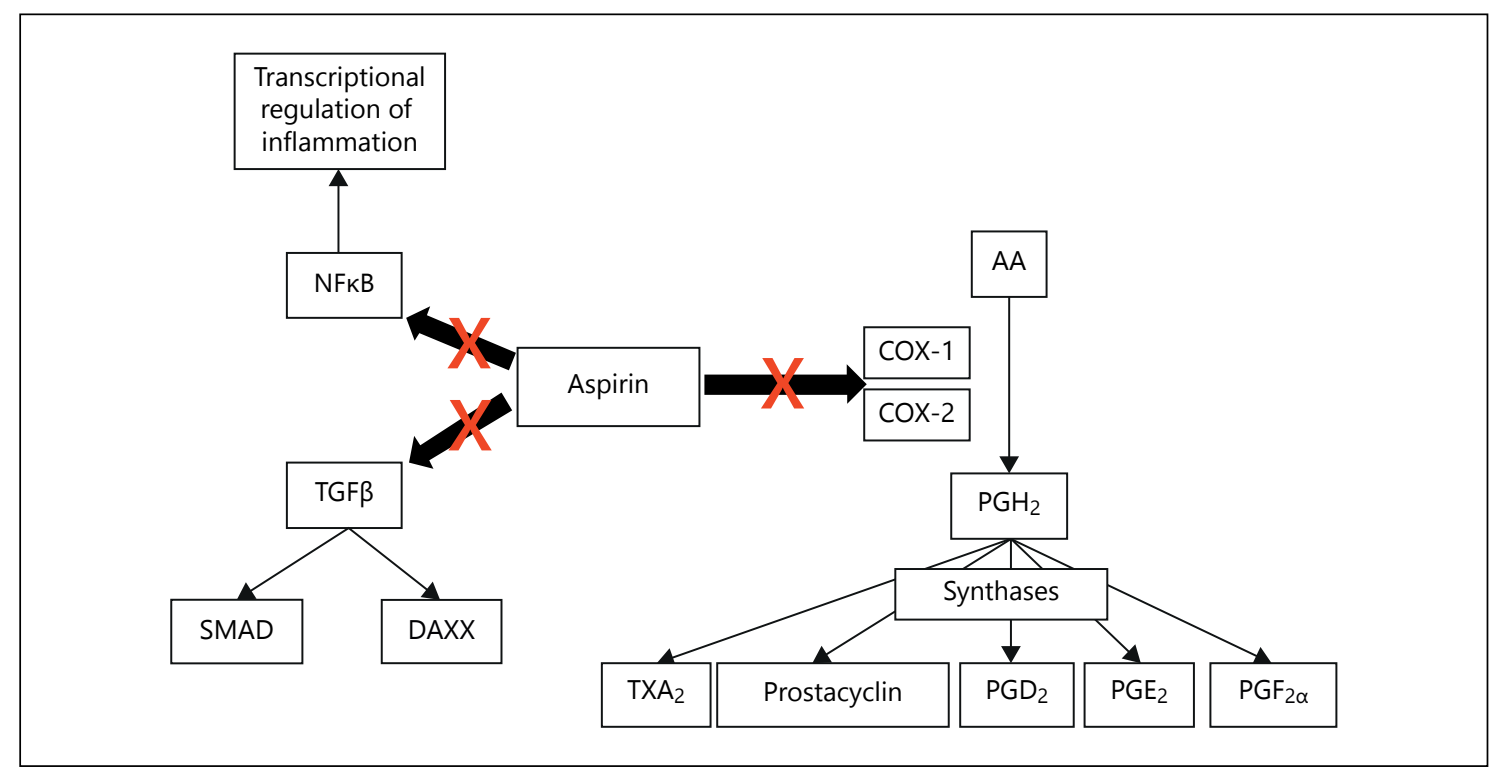

Fig. 1. Anti-inflammatory and antithrombotic aspirin cascade.

has evolved significantly over the past two decades, the rate of hospital admission for SAH has not decreased [3]. The rate of SAH-related mortality is approximately $50 \%$ with approximately one half of survivors suffering from long-term neurocognitive deficits and one third requiring permanent assistance [4-6]. Therefore, the most effective method for improving the currently dismal outcomes associated with aneurysm hemorrhage is to prevent rupture [7].

In patients without a known family history of aneurysms, screening noninvasive angiography is impractical. Unfortunately, a reliable serum test for aneurysm detection in the general population is yet to be validated. Therefore, our focus remains on treating those patients with known aneurysms to prevent the catastrophic morbidity, which frequently accompanies rupture. The most direct approach for preventing aneurysm rupture is obliteration via either an endovascular or microsurgical approach. However, despite advances in endovascular and microsurgical techniques, the morbidity and mortality associated with the treatment of unruptured aneurysms are not inconsequential $[8,9]$. The morbidity and mortality from rupture are still significant in aneurysm patients who are deemed low risk for rupture, high risk for rupture, or both. Therefore, noninvasive therapies for aneurysm stabilization and regression are attractive targets of current preclinical research efforts [10].

Inflammation plays a key role in propagating aneurysm destabilization toward eventual rupture [11, 12].
Recent studies have demonstrated that aspirin (acetylsalicyclic acid), a commonly used antiplatelet drug, may decrease the incidence of aneurysmal SAH by counteracting pro-inflammatory pathways and stabilizing aneurysm walls [13-15]. The goal of this review was to critically analyze the biological mechanisms by which aspirin attenuates inflammation and its potential role in abrogating pathways leading to formation, progression, and rupture of intracranial aneurysms.

\section{Potential Therapeutic Role of Aspirin in Cerebral Aneurysms}

Because endovascular and microsurgical treatment of intracranial aneurysms can be associated with significant morbidity and in some cases may be cost-prohibitive, noninvasive and affordable pharmacological therapies are needed. Inflammation is thought to play a central role in the pathogenesis of intracranial aneurysm rupture [10, 11]. Aspirin has emerged as a promising candidate for noninvasive treatment of intracranial aneurysms, largely through its anti-inflammatory effects $[13,14,16-20]$ (fig. 1). Studies have shown that aspirin may act on several constituents of the inflammatory response that have been demonstrated to play a key role in the pathogenesis of intracranial aneurysms (table 1).

Endothelial cell dysfunction is an early step in aneurysm pathogenesis. Macrophages and vascular smooth 
Table 1. Potential molecular targets of aspirin in cerebral aneurysm pathophysiology

\begin{tabular}{|c|c|}
\hline Target & Biochemical physiology \\
\hline Cyclooxygenase-1 (COX-1) & $\begin{array}{l}\text { Constitutively expression isoforms of COX, which catalyze the conversion of AA to } \\
\mathrm{PGH}_{2} \text {. }\end{array}$ \\
\hline Cyclooxygenase-2 (COX-2) & $\begin{array}{l}\text { Inducible isoform of COX, which is expressed only in pro-inflammatory conditions. } \\
\text { Similar to COX-1, it also promotes the conversion of AA to } \mathrm{PGH}_{2} \text {. }\end{array}$ \\
\hline Arachidonic acid (AA) & $\begin{array}{l}\text { A polyunsaturated fatty acid abundantly present in cellular membranes. AA is } \\
\text { generated from membrane phospholipids by phospholipases } \mathrm{A}_{2} \text { and C. AA is a } \\
\text { critically important intermediate product and is a precursor molecule to multiple } \\
\text { downstream eicosanoids. }\end{array}$ \\
\hline Prostaglandin $\mathrm{H}_{2}\left(\mathrm{PGH}_{2}\right)$ & $\begin{array}{l}\text { Another key intermediate product, which is generated from AA by COX-1 and } \\
\mathrm{COX}-2 . \mathrm{PGH}_{2} \text { is converted by several distinct enzymes to downstream eicosanoids } \\
\text { with both pro- and anti-inflammatory effects. }\end{array}$ \\
\hline Prostacyclin $\left(\mathrm{PGI}_{2}\right)$ & $\begin{array}{l}\text { A physiologic antagonist of } \mathrm{TXA}_{2} \text { derived from } \mathrm{PGH}_{2} \text {. Prostacyclin promotes } \\
\text { vasodilation, decreases smooth muscle cell and fibroblast proliferation, increases } \\
\text { pro-apoptotic signaling, decreases platelet aggregation adherence to vessel walls, } \\
\text { inhibits pro-inflammatory signaling by decreasing levels of IL-1 and IL- } 6 \text {, promotes } \\
\text { anti-inflammatory signaling by increasing levels of IL-10, inhibits angiogenesis by } \\
\text { lowering levels of VEGF, and promotes extracellular matrix remodeling by lower levels } \\
\text { of TGF- } \beta \text {. }\end{array}$ \\
\hline Prostaglandin $\mathrm{D}_{2}\left(\mathrm{PGD}_{2}\right)$ & $\begin{array}{l}\text { A pro-inflammatory eicosanoid derived from } \mathrm{PGH}_{2} \cdot \mathrm{PGD}_{2} \text { is produced by activated } \\
\text { mast cells and results in recruitment of Type } 2 \mathrm{~T} \text { helper cells, eosinophils, and } \\
\text { basophils, which further propagate the inflammatory cascade. }\end{array}$ \\
\hline Transforming growth factor- $\beta$ (TGF- $\beta$ ) & $\begin{array}{l}\text { An anti-inflammatory cytokine, which promotes apoptosis via activation of the SMAD } \\
\text { or DAXX signaling pathways. TGF- } \beta \text { also potentiates the immunosuppressive effects } \\
\text { of regulatory T cells. }\end{array}$ \\
\hline $\begin{array}{l}\text { Nuclear factor } \kappa \text {-light-chain-enhancer of } \\
\text { activated B cells (NF- } \kappa \text { B) }\end{array}$ & $\begin{array}{l}\text { A transcription factor, which is activated by inflammation and regulates the expression } \\
\text { of many genes involved in the inflammatory cascade, including cytokines, immune } \\
\text { receptors, and cell adhesion molecules. NF- } \mathrm{kB} \text { also regulates the differentiation and } \\
\text { proliferation of various immune cells. }\end{array}$ \\
\hline
\end{tabular}

muscle cells are essential elements of the cascade of events leading to aneurysm rupture [21]. Numerous inflammatory molecules, such as nuclear factor kappa B (NF-kB), interleukins, tumor necrosis factor- $\alpha$ (TNF- $\alpha$ ), and monocyte chemoattractant protein-1 (MCP-1), also play an integral role in the inflammatory reaction in aneurysm walls [10]. The inflammatory cascade leads to the release of matrix metalloproteinases (MMP) by macrophages, with degradation of the extracellular matrix, weakening of the arterial wall, and ultimately aneurysm rupture. There is data to suggest that aspirin inhibits TNF- $\alpha$ mediated expression of NF- $\kappa \mathrm{B}$, induction of adhesion 


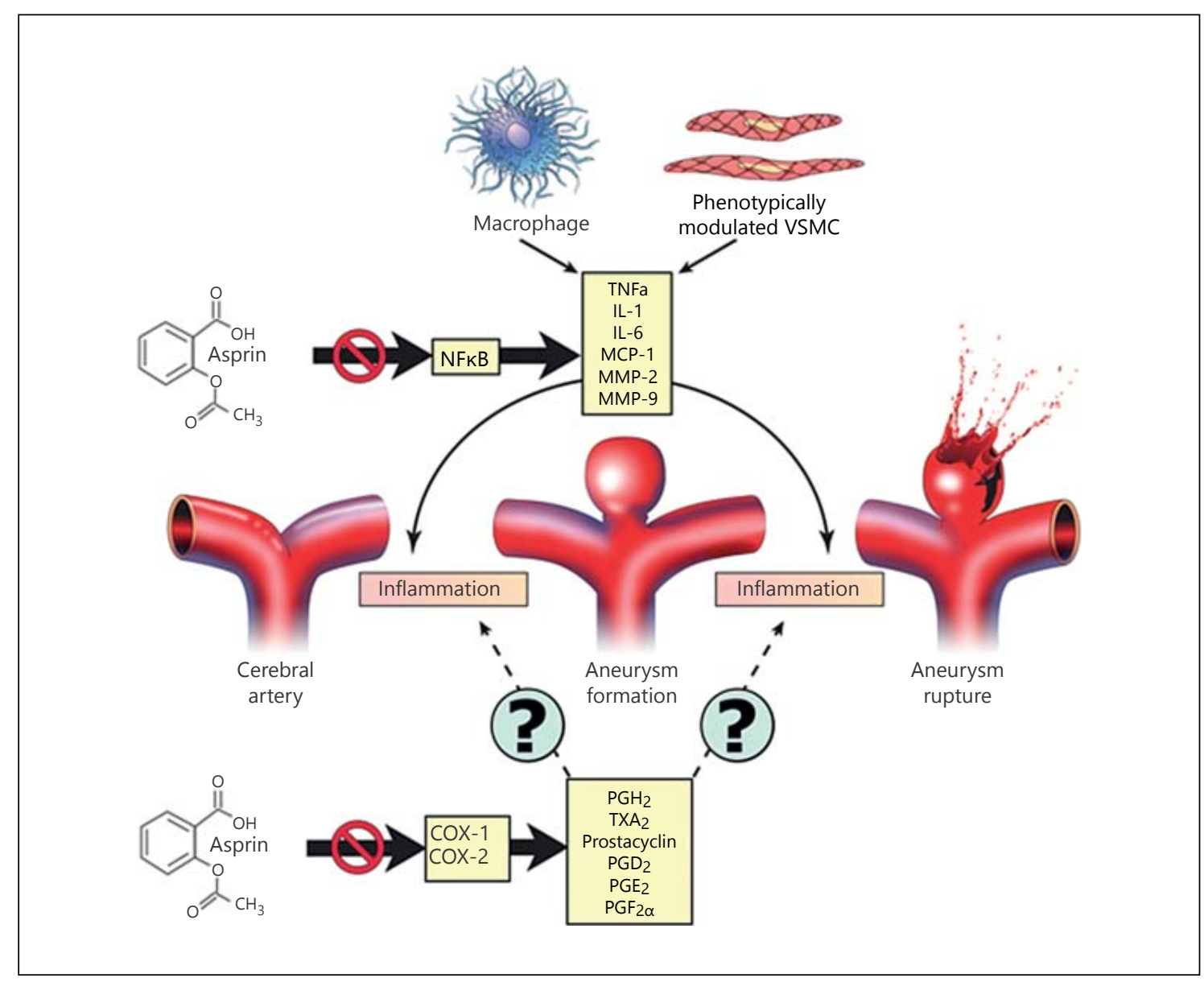

Fig. 2. Potential role of aspirin in the alteration of cerebral aneurysm pathophysiology.

molecules, and adhesion of monocytes in endothelial cells [22]. It also blocks the expression of TNF- $\alpha$ release in smooth muscle cells and macrophages [23, 24]. Additionally, aspirin directly inhibits MMP-2 and MMP-9 expression in macrophages, and may also induce the expression of tissue inhibitors of metalloproteinases (TIMPs) [25]. These molecular mediators have been found to be critical to aneurysm formation and rupture in animal models and have been found to be upregulated in human cerebral aneurysms (fig. 2) [10].

In an animal model of cerebral aneurysm formation and rupture, we have found aspirin to have a protective effect. Mice treated with Celebrex, the selective COX-2 inhibitor and COX-2 knockout mice had a decreased incidence of aneurysm formation and rupture. There was no alteration in aneurysm formation or rupture in mice treated with SC560 (COX-1 inhibitor) or in COX-1 knockout mice (unpublished). This provides further support for the potential role of aspirin in inhi- bition of COX-2 in cerebral aneurysm formation and rupture and provides further support for human clinical trials.

\section{Evidence of the Protective Role of Aspirin in Human SAH}

COX-2 is an attractive target in human cerebral aneurysms. We have previously found that the walls of ruptured human intracranial aneurysms have higher levels of COX-2 and microsomal prostaglandin E2 synthase 1 (mPGES-1), both of which are known to be inhibited by aspirin [17]. Based on this prior work, we have found in a randomized trial that patients harboring unruptured intracranial aneurysms who were treated with aspirin (81 mg daily for 3 months) had attenuated expression of COX-2, mPGES-1, and macrophages in aneurysm walls after 3 months of aspirin therapy [14]. Additionally, we 


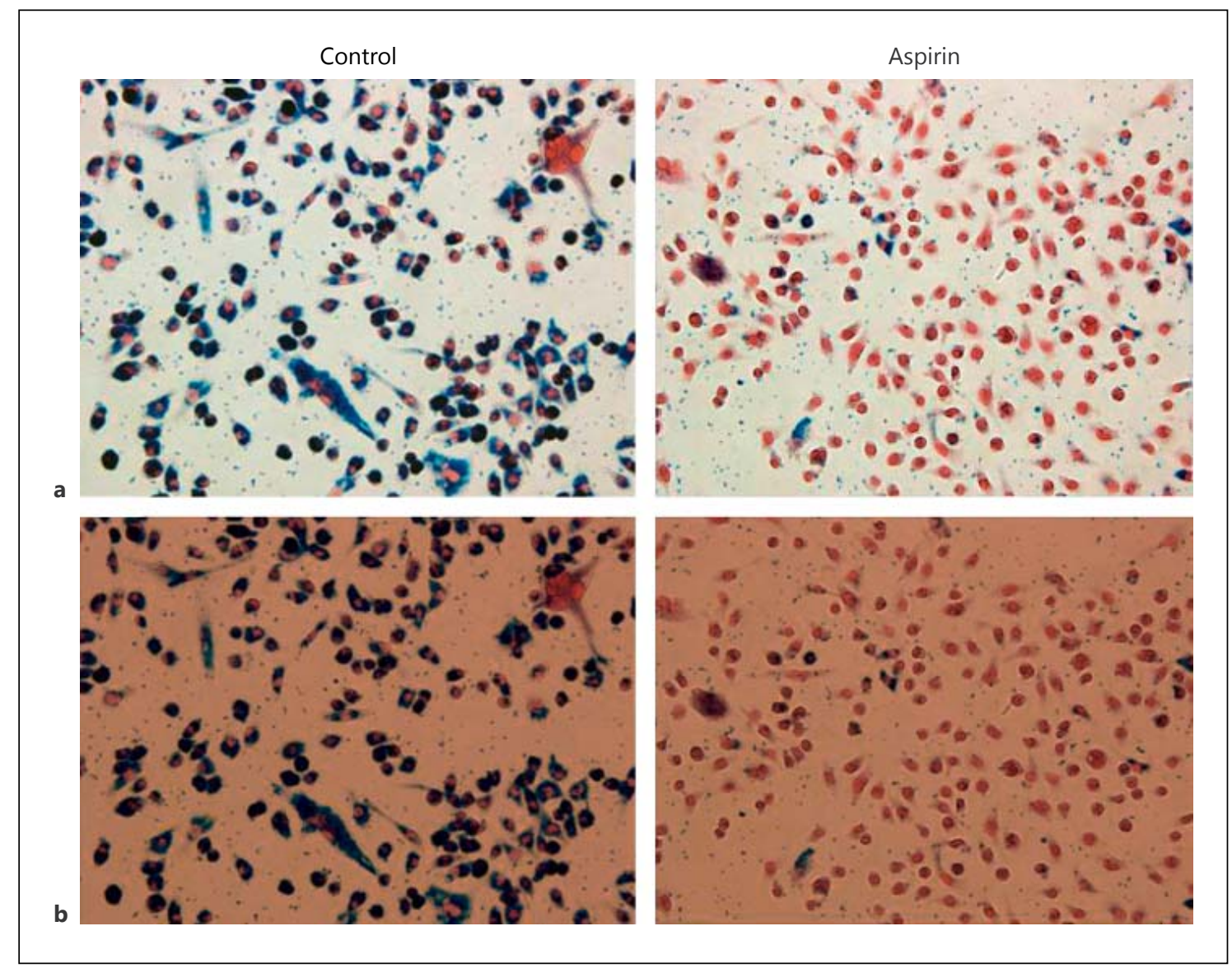

Fig. 3. Macrophages (a) and ferumoxytol (b) uptake. Macrophages incubated with and ferumoxytol and lipopolysaccharide were treated with either aspirin or vehicle for $24 \mathrm{~h}$. Immunohistochemistry demonstrates decreased uptake of ferumoxytol by the macro- phages treated with aspirin. This supports the findings on ferumoxytol-MRI where patients treated with aspirin had decreased intensity of the signal on the T2 sequence suggesting decreased inflammation in the aneurysm wall. have assessed local circulating expression of chemokines and COX-2 in relation to cerebral aneurysms. Inflammatory chemokines known to play a role in cerebral aneurysm formation were increased locally in blood samples taken from within aneurysm domes as compared to peripheral blood sample controls [26]. Similarly, in patients who underwent endovascular treatment of cerebral aneurysms, blood was collected from the dome of the aneurysm and femoral arteries. At this time, a stent was placed and patients were started on aspirin. After allowing time for the stent to heal and endothelialize, the patients underwent aneurysm coiling one month later while still on aspirin. The assessment of peripheral femoral artery blood as compared to intra-aneurysmal blood demonstrated increased expression of COX-2 within leukocytes, which normalized following initiation of aspirin (unpublished; fig. 3). These findings provided evidence that as- pirin decreased the inflammatory reaction in aneurysm walls and may potentially be used as a therapeutic agent to prevent intracranial aneurysm growth and rupture.

Further evidence of a protective role of aspirin against intracranial aneurysm rupture comes from further human clinical studies. We have previously found that in a nested case-control study from the International Study of Unruptured Intracranial Aneurysms (the largest study to date on the natural history of intracranial aneurysms) found that patients taking aspirin at least three times weekly had a significantly lower risk of SAH (OR, 0.27; 95\% CI, 0.11-0.67; $\mathrm{p}=0.03$ ) compared with those who never took aspirin [13]. There was a linear association between aspirin intake (time per week) and the incidence of aneurysm rupture.

These findings were replicated by another large, welldesigned study by García-Rodríguez and colleagues [27] 
that examined the relationship between the use of antiplatelets/warfarin and the risk of hemorrhagic stroke. Compiling data from 1,797 patients with intracerebral hemorrhage, 1,340 patients with subarachnoid hemorrhage (SAH), and 10,000 selected controls, the authors determined the risk of hemorrhagic stroke in users and nonusers of antiplatelets after adjusting for demographic/ lifestyle factors and comorbidities. Importantly, chronic low dose aspirin therapy was found to have a protective effective against $\mathrm{SAH}$, especially in patients on long-term aspirin therapy ( $>3$ years). Equally important was the finding that aspirin did not increase the risk of intracerebral hemorrhage.

Some authors believe that the protective effect of aspirin against aneurysm rupture is related to its antiplatelet effects since inhibition of platelet activation reduces endothelial injury, mural thrombus formation, and subsequent aneurysm wall inflammation. While this could be a possible mechanism, a recent study [27] showing no protective effect for clopidogrel (a more potent antiplatelet agent) against SAH argues against this hypothesis. Furthermore, studies have found that aspirin has not increased the bleeding complications following aneurysm rupture and may have a beneficial overall effect on outcome. In a review of 747 consecutive patients with cerebral aneurysms, Gross et al. [15] compared the rate of hemorrhagic presentation in those taking aspirin and those who were not. They found that the rate of hemorrhagic presentation was significantly lower in patients taking aspirin ( 28 vs. $40 \%, \mathrm{p}=0.01$ ). The authors also compared factors like demographics, aneurysm characteristics, clinical and radiographic grades, vasospasm, and outcome at 1 year between patients with aneurysmal SAH taking aspirin on presentation and those who were not. The 2 groups were comparable with regard to baseline characteristics and there was no difference in the rate of delayed cerebral ischemia. The predictors of poor outcome were increasing age, higher Hunt and Hess grades, and hypertension, whereas aspirin use was not associated with poor outcome. This is a crucial observation because neurosurgeons tend to assume that aspirin would worsen the bleed and adversely affect the outcome of patients with SAH. A randomized controlled trial that compared the risk of delayed cerebral ischemia in SAH patients taking aspirin or not did not find a beneficial effect of aspirin in this respect [28]. However, it should be noted that aspirin was not associated with worse outcomes. Moreover, there was a $21 \%$ reduction in the risk of poor outcome with aspirin therapy, although the difference never reached statistical significance [28].

Aspirin and Cerebral Aneurysms
Overall, because aspirin may prevent aneurysm rupture and does not have a negative effect on outcomes in $\mathrm{SAH}$ patients, it is reasonable to consider aspirin as one of the treatments of intracranial aneurysms to go along with endovascular therapy and surgical clipping.

\section{Aspirin and Innovations in Molecular Imaging in Cerebral Aneurysms}

Ferumoxytol-enhanced MRI is a technique that is recently being investigated. Ferumoxytol is a member of the class of ultrasmall superparamagnetic iron oxide and is approved for treatment for iron deficiency anemia in patients with chronic renal failure. It is also used as an intravascular contrast agent and an inflammatory marker when imaging is delayed because it is cleared by macrophages (usually within 24-72 h) [29].

We assessed in a small study whether ferumoxytol-enhanced MRI can be used to noninvasively measure the effects of aspirin on intracranial aneurysms [16-18, 30]. Five patients with intracranial aneurysms underwent baseline imaging using ferumoxytol-enhanced MRI before they received $325 \mathrm{mg}$ aspirin daily. After 3 months of treatment, imaging studies revealed decreased signal intensity in aneurysm walls compared to the baseline images in all 5 patients. These results suggested that aspirin attenuates inflammation in aneurysm walls and that ferumoxytol-enhanced MRI allows the possibility of imaging the effect of aspirin on intracranial aneurysms.

We investigated in a randomized controlled trial the effects of aspirin on inflammatory cells and molecules in the wall of human cerebral aneurysms [14]. We randomized 11 patients with unruptured intracranial aneurysms into aspirin-treated ( $81 \mathrm{mg}$ daily) $(\mathrm{n}=6)$ and untreated (control) groups $(\mathrm{n}=5)$. We obtained imaging of the aneurysms at baseline using ferumoxytol-enhanced MRI to estimate uptake by macrophages and after three months before microsurgical clipping (fig. 4). The signal intensity in patients on aspirin was remarkably decreased in the wall of cerebral aneurysms on ferumoxytol-enhanced MRI, whereas in the control group, the signal intensity did not change. Importantly, the findings of ferumoxytolenhanced MRI were consistent with immunostaining in that the expression of cyclooxygenase-1, microsomalprostaglandin-E2 synthase-1, and macrophages was significantly lower in the aspirin group than in the control group.

To further assess the role of aspirin in this process, incubated macrophages were stimulated with lipopolys- 


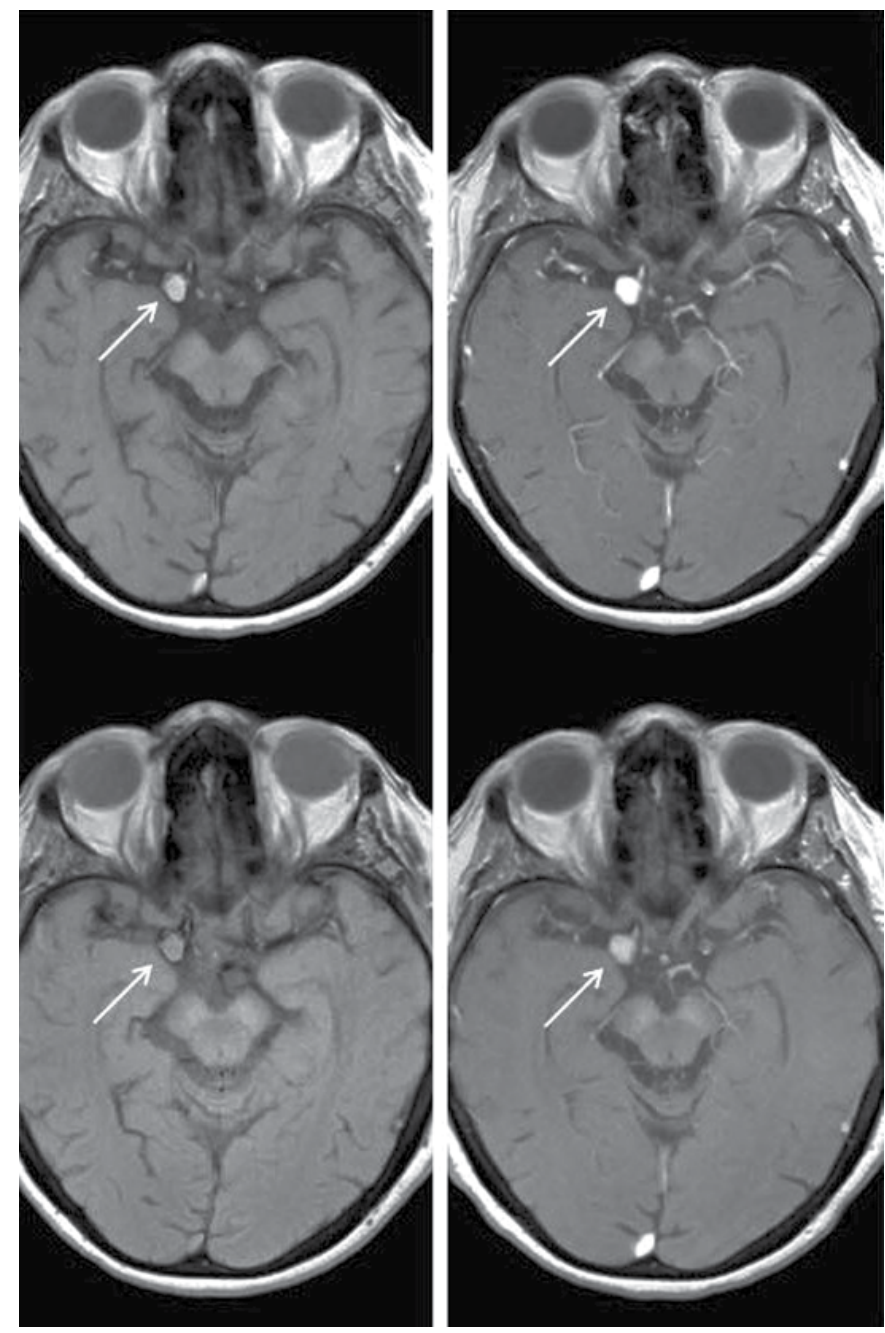

Fig. 4. Ferumoxytol MRI before and after aspirin treatment. Top row: MRI demonstrates right posterior communicating artery aneurysm (arrow) $24 \mathrm{~h}$ post infusion of ferumoxytol and $72 \mathrm{~h}$ post ferumoxytol infusion, demonstrating increased signal within the aneurysm. Bottom row: Three months after aspirin treatment, MRI $24 \mathrm{~h}$ post ferumoxytol infusion and $72 \mathrm{~h}$ post ferumoxytol demonstrate attenuated signal within the aneurysm.

caracide and ferumoxytol and then were treated with aspirin versus vehicle. Immunohistochemistry demonstrated that macrophages treated with aspirin had a marked decrease of uptake of ferumoxytol. This supports the findings on ferumoxytol-MRI where patients treated with aspirin had decreased intensity of the signal on the T2 sequence, suggesting decreased inflammation in the aneurysm wall. These studies provide further evidence that aspirin may attenuate the inflammatory process in the wall of human cerebral aneurysms and that ferumoxytol-enhanced MRI is a reliable technique to monitor the effects of aspirin on aneurysms. Overall, with further refinement in technique and quantification of signal intensity, ferumoxytol-enhanced MRI may be used to identify patients who respond to aspirin therapy versus those who will require more invasive intervention.

\section{The Effect of Aspirin on the Coagulation Cascade}

Aspirin does not directly act on the coagulation cascade but instead causes a permanent defect in platelet function [31]. Aspirin irreversibly inhibits the two primary isoforms of the enzyme cyclooxygenase (COX), COX-1 and COX-2, which catalyze the synthesis of multiple eicosanoids from arachidonic acid (AA) (fig. 1). COX-1 converts AA to prostaglandin $\mathrm{H}_{2}\left(\mathrm{PGH}_{2}\right)$, which is then converted to thromboxane $\mathrm{A}_{2}\left(\mathrm{TXA}_{2}\right)$ in activated platelets by thromboxane synthase. $\mathrm{TXA}_{2}$ propagates a positive feedback loop by binding to the G-protein coupled $\mathrm{TXA}_{2}$ receptor on other platelets, thereby inducing activation and recruiting them to the site of injury. Recruitment of activated platelets allows further cross-linking via fibrin and expansion of the platelet plug. In a biochemical pathway parallel to that of $\mathrm{TXA}_{2}$ synthesis, $\mathrm{PGH}_{2}$ is also converted to prostacyclin, otherwise known as prostaglandin $\mathrm{I}_{2}\left(\mathrm{PGI}_{2}\right)$, by COX-2 in endothelial cells [32]. Prostacyclin is a physiological antagonist to the $\mathrm{TXA}_{2}$, causing vasodilation and discouraging thrombosis. The balance between $\mathrm{TXA}_{2}$ and prostacyclin regulates the platelet response to vascular endothelial injury.

Aspirin indirectly inhibits the coagulation cascade by decreasing the formation of $\mathrm{TXA}_{2}$. The mechanism by which aspirin causes functional platelet inhibition is the prevention of platelet aggregation and cross-linking via fibrin. The antiplatelet effects of aspirin make it useful in preventing thromboembolic events associated with a variety of vascular diseases, but they may be also responsible for the hemorrhagic complications [33].

\section{Aspirin and Inhibition of Inflammation}

In addition to its efficacy as an antiplatelet drug, aspirin is also a powerful inhibitor of inflammation. The classically described pathway through which aspirin ameliorates the inflammatory response is via $\mathrm{COX}$ inhibition, thereby decreasing the conversion of AA to $\mathrm{PGH}_{2}$. $\mathrm{PGH}_{2}$ is not only converted to $\mathrm{TXA}_{2}$ and prostacyclin but also to prostaglandins $\mathrm{D}_{2}\left(\mathrm{PGD}_{2}\right), \mathrm{E}_{2}\left(\mathrm{PGE}_{2}\right)$, and $\mathrm{F}_{2 \alpha}\left(\mathrm{PGF}_{2 \alpha}\right)$, 
all of which act by autocrine- or paracrine-signaling mechanisms through their respective G-protein-coupled prostaglandin receptors [34]. Furthermore, $\mathrm{TXA}_{2}$ and prostacyclin affect more than just the regulation of platelet aggregation; they also play important roles in the inflammatory cascade. The combined effect of the $\mathrm{PGH}_{2}$ derived prostaglandins in humans is the enrichment of pro-inflammatory signaling.

COX-1 is constitutively expressed, while COX-2 expression is inducible by inflammation. However, both isozymes have been shown to contribute to the inflammatory response [35]. Human studies suggest that COX-1 mediates the initial acute inflammatory response, while COX-2 induction occurs several hours after ictus perpetuates it [36]. A multitude of preclinical animal models have been used to study the effects of both COX-1 and COX-2 genetic and biochemical inactivation with knockout models and pharmacological inhibitors [37]. COX-2 has been demonstrated to possess dual pro- and anti-inflammatory roles by propagating the initial phase of inflammation but aiding in its resolution in later phases [38]. The delineation between which synthetic products of COX-2 contribute to each of these two functions is yet to be made.

In addition to its COX-dependent anti-inflammatory mechanisms, literature supporting COX-independent mechanisms by which aspirin suppresses inflammation is beginning to accumulate. The mechanisms underlying the COX-independent effects of aspirin are not well understood, but preliminary data suggests that aspirin may reduce signaling through the transforming growth factor- $\beta$ (TGF- $\beta$ ) signaling pathway [39]. Other studies have implicated aspirin in blockade of the nuclear factor $\kappa$-light-chain-enhancer of activated B cells (NF- $\kappa \mathrm{B})$ transcriptional activation pathway [40]. The contribution of COX-dependent versus COX-independent mechanisms to the physiological anti-inflammatory impact of aspirin remains unknown.

\section{Aspirin in Peripheral Vascular Disease, Atherosclerosis, and Aortic Aneurysms}

Although the beneficial role of aspirin in a number of disease processes is due to its antithrombotic effects, inhibition of inflammation is a critical element. Aspirin has also been found to be beneficial in the inhibition of cancer [41-43]. Pooled analysis of randomized controlled trials showed that daily aspirin use reduces the risk of colorectal cancer by $24 \%$ and associated mortality by $35 \%$ after
8-10 years [44, 45]. Another meta-analysis revealed that daily aspirin use led to a $21 \%$ reduction in the risk of all cancer death, including colorectal cancer [44].

Aspirin is the mostly widely prescribed antiplatelet medication for the secondary prevention of cerebral and myocardial ischemia secondary to vascular disease [46]. Atherosclerosis is a chronic inflammatory process by which vascular endothelial injury results in lipid accumulation in the subendothelial layer of vessel walls resulting in plaque formation. Atherosclerosis is the primary pathobiological process underlying the leading causes of mortality worldwide [47]. The clinical endstage event of atherosclerosis-related diseases depends on the location of the plaque; atherosclerosis of the coronary arteries leads to myocardial infarction (MI), atherosclerosis of the extracranial and intracranial carotid and vertebrobasilar circulations lead to stroke, and atherosclerosis of the peripheral arteries leads to vascular claudication and limb ischemia. Additionally, atherosclerosis contributes to the development of aortic and cerebral aneurysms $[48,49]$.

For patients with coronary artery disease (CAD), extracranial carotid atherosclerotic disease (ECAD), and intracranial atherosclerotic disease (ICAD), there is class I evidence that aspirin is clinically effective and cost-effective for secondary prevention of stroke and MI [46, 50]. By discouraging platelet aggregation, aspirin reduces the thromboembolic complications associated with CAD, ECAD, and ICAD [51-53]. A trial of patients with recent transient ischemic attack (TIA) or minor ischemic stroke, demonstrated that treatment with aspirin and clopidogrel, which inhibits platelet aggregation by a mechanism distinct from aspirin, affords further protection from ischemic stroke without an increase in hemorrhagic complications compared to aspirin monotherapy [54]. For stroke prevention in symptomatic ICAD, aspirin has been demonstrated to possess equivalent efficacy but superior safety compared to warfarin [55]. A multicenter, prospective, randomized trial comparing endovascular stenting to aggressive medical management for symptomatic, severe ICAD demonstrated superior outcomes in the medical management cohort with a dual antiplatelet regimen including aspirin and clopidogrel [50].

For patients with peripheral vascular disease (PVD), the benefit of aspirin in reducing the risk of stroke or MI is controversial [56, 57]. A meta-analysis of 18 prospective, randomized trials including over 5,000 patients demonstrated that aspirin decreased the risk of cardiovascular events, which was not statistically significant 
(relative risk $0.88, \mathrm{p}=0.13$ ) but significantly decreased the risk of nonfatal stroke (relative risk $0.66, p=0.02$ ) [58]. There was no significant effect of aspirin on all-cause mortality $(p=0.85)$ or major hemorrhage $(p=0.98)$. Despite the lack of substantial evidence supporting its benefit for PVD patients, aspirin remains the first-line therapy for stroke and MI prevention due to its relatively favorable benefit to risk profile [59].

In a manner similar to cerebral aneurysms, inflammation is believed to play a crucial role in the development of AAAs [48]. T regulatory cells, which are natural suppressors of the immune response and inflammation, have been shown to regulate AAA formation and decrease their rupture [60]. Intraluminal mural thrombus (ILT) development, a frequent occurrence in AAAs, accelerates their progression and predisposes them to rupture by decreasing oxygen diffusion to the aneurysm wall thereby triggering a hypoxia-induced inflammatory response, which causes focal weakening of the wall [61]. Aspirin is believed to limit the enlargement and decrease the rupture risk of AAAs by acting through antiplatelet mechanisms to reduce ILT formation and by anti-inflammatory mechanisms to decrease the immune response to vascular endothelia injury and oxidative stress [62]. However, there is currently a paucity of clinical data supporting the protective effects of aspirin on AAA progression and rupture.

\section{Conclusion}

Aspirin is a beneficial therapy in many disease states due to its potent anti-inflammatory mechanisms. Currently, there are no pharmacological therapies for patients with cerebral aneurysms, but inflammation is a critical element of cerebral aneurysm pathophysiology. In animal models, treatment with aspirin and the genetic inactivation of COX-2 decrease aneurysm formation and rupture. Treatment of human patients with aspirin also decreases the expression of COX-2 within cerebral aneurysms and markers of intra-aneurysmal inflammation. Novel molecular imaging with ferumoxytol-enhanced MRI may allow the identification of patients at increased risk for aneurysm rupture and assess response to aspirin therapy.

Evidence reviewed supports the continuation of aspirin in all patients newly diagnosed with aneurysm who are already taking aspirin. It is also reasonable to start aspirin in patients with unruptured aneurysms in whom observation is elected. Aspirin could be prescribed in pa- tients who do not meet criteria for invasive treatment (advanced age; small aneurysm; significant comorbidities) or in those who elect for conservative treatment. Aspirin could be added as an adjunct to decrease the inflammatory reaction in aneurysm walls and prevent aneurysm recurrence but this would require further investigation.

Aspirin is an affordable and widely available drug especially in developing countries that lack resources for surgical or endovascular management of aneurysms. Aspirin is also a safe drug that has several other health benefits including prevention of myocardial infarction, ischemic stroke, and colorectal cancer. Clinicians and patients are also very familiar with aspirin, its dosing regimen and side effects, which facilitates its use for the prevention of SAH.

Aspirin has been found to be safe in patients harboring cerebral aneurysms, and clinical studies provide evidence that it may decrease the overall rate of rupture. Additionally, it is easily accessible and inexpensive. Future trials are indicated to determine the effect of aspirin on aneurysm progression and rupture. Currently, a multicenter randomized clinical trial is planned to assess the role of aspirin in inhibition of aneurysm rupture. Patients older than 65 years of age with aneurysms $<7 \mathrm{~mm}$ deemed at low risk of rupture, high risk of treatment, or both would be assigned to current best medical therapy with our without aspirin. Additionally, a subcohort of patients with unruptured brain aneurysms will undergo the longitudinal follow-up to assess whether aneurysms that grow or rupture exhibit increased inflammation as demonstrated on ferumoxytol-enhanced MRI.

\section{Acknowledgment}

The authors would like to thank Philip Cohen for his elegant work on the illustrations.

\section{Funding}

This work was supported by the National Institute of Neurological Disorders and Stroke (R03NS079227-01A1) and (K08NS 082363-01A1) to DMH.

\section{Disclosure Statement}

None declared. 


\section{References}

1 Suarez JI, Tarr RW, Selman WR: Aneurysmal subarachnoid hemorrhage. N Engl J Med 2006;354:387-396.

-2 van Gijn J, Rinkel GJ: Subarachnoid haemorrhage: diagnosis, causes and management. Brain 2001;124:249-278.

-3 Rincon F, Rossenwasser RH, Dumont A: The epidemiology of admissions of nontraumatic subarachnoid hemorrhage in the United States. Neurosurgery 2013;73:217-222; discussion 222-223.

4 Hop JW, Rinkel GJ, Algra A, van Gijn J: Casefatality rates and functional outcome after subarachnoid hemorrhage: a systematic review. Stroke 1997;28:660-664.

$\checkmark 5$ Mayer SA, Kreiter KT, Copeland D, et al: Global and domain-specific cognitive impairment and outcome after subarachnoid hemorrhage. Neurology 2002;59:1750-1758.

-6 Brilstra EH, Rinkel GJ, van der Graaf Y, et al: Quality of life after treatment of unruptured intracranial aneurysms by neurosurgical clipping or by embolisation with coils. A prospective, observational study. Cerebrovasc Dis 2004;17:44-52.

7 Steiner T, Juvela S, Unterberg A, Jung C Forsting M, Rinkel G: European Stroke Organization guidelines for the management of intracranial aneurysms and subarachnoid haemorrhage. Cerebrovasc Dis 2013;35:93112.

8 Unruptured intracranial aneurysms - risk of rupture and risks of surgical intervention. International study of unruptured intracranial aneurysms investigators. N Engl J Med 1998; 339:1725-1733.

9 Wiebers DO, Whisnant JP, Huston J 3rd, et al: Unruptured intracranial aneurysms: natural history, clinical outcome, and risks of surgical and endovascular treatment. Lancet 2003. 362:103-110.

10 Chalouhi N, Ali MS, Jabbour PM, et al: Biology of intracranial aneurysms: role of inflammation. J Cereb Blood Flow Metab 2012;32: 1659-1676.

11 Chalouhi N, Ali MS, Starke RM, et al: Cigarette smoke and inflammation: role in cerebral aneurysm formation and rupture. Mediators Inflamm 2012;2012:271582.

12 Ali MS, Starke RM, Jabbour PM, et al: TNF- $\alpha$ induces phenotypic modulation in cerebral vascular smooth muscle cells: implications for cerebral aneurysm pathology. J Cereb Blood Flow Metab 2013;33:1564-1573.

13 Hasan DM, Mahaney KB, Brown RD Jr, et al: Aspirin as a promising agent for decreasing incidence of cerebral aneurysm rupture. Stroke 2011;42:3156-3162.

14 Hasan DM, Chalouhi N, Jabbour P, et al: Evidence that acetylsalicylic acid attenuates inflammation in the walls of human cerebral aneurysms: preliminary results. J Am Heart Assoc 2013;2:e000019.

15 Gross BA, Rosalind Lai PM, Frerichs KU, Du $\mathrm{R}$ : Aspirin and aneurysmal subarachnoid hemorrhage. World Neurosurg 2014;82: 28 van den Bergh WM; MASH Study Group, Al$1127-1130$

16 Hasan D, Chalouhi N, Jabbour P, et al: Early change in ferumoxytol-enhanced magnetic resonance imaging signal suggests unstable human cerebral aneurysm: a pilot study. Stroke 2012;43:3258-3265.

17 Hasan D, Hashimoto T, Kung D, Macdonald RL, Winn HR, Heistad D: Upregulation of cyclooxygenase-2 (COX-2) and microsomal prostaglandin E2 synthase-1 (mPGES-1) in wall of ruptured human cerebral aneurysms: preliminary results. Stroke 2012;43:19641967.

18 Hasan DM, Chalouhi N, Jabbour P, Magnotta VA, Kung DK, Young WL: Imaging aspirin effect on macrophages in the wall of human cerebral aneurysms using ferumoxytol-enhanced MRI: preliminary results. J Neuroradiol 2013;40:187-191.

19 Chalouhi N, Jabbour P, Hasan D, Starke RM: Aspirin for prevention of subarachnoid hemorrhage: the stage is set for a randomized controlled trial. Neurosurgery 2014;74:E147E148.

20 Chalouhi N, Jabbour P, Starke RM, Hasan DM: Aspirin for prophylaxis against cerebral aneurysm rupture. World Neurosurg 2013; 81:e2-e3.

21 Starke RM, Chalouhi N, Ding D, et al: Vascular smooth muscle cells in cerebral aneurysm pathogenesis. Transl Stroke Res 2014;5:338346.

22 Weber C, Erl W, Pietsch A, Weber PC: Aspirin inhibits nuclear factor-kappa $B$ mobilization and monocyte adhesion in stimulated human endothelial cells. Circulation 1995;91: 1914-1917.

23 Shackelford RE, Alford PB, Xue Y, Thai SF, Adams DO, Pizzo S: Aspirin inhibits tumor necrosis factoralpha gene expression in $\mathrm{mu}$ rine tissue macrophages. Mol Pharmacol 1997;52:421-429.

24 Sánchez de Miguel L, de Frutos T, GonzálezFernández F, et al: Aspirin inhibits inducible nitric oxide synthase expression and tumour necrosis factor-alpha release by cultured smooth muscle cells. Eur J Clin Invest 1999; 29:93-99.

25 Hua Y, Xue J, Sun F, Zhu L, Xie M: Aspirin inhibits MMP-2 and MMP-9 expressions and activities through upregulation of PPARalpha/ gamma and TIMP gene expressions in oxLDL-stimulated macrophages derived from human monocytes. Pharmacology 2009;83: $18-25$.

26 Chalouhi N, Points L, Pierce GL, Ballas Z, Jabbour P, Hasan D: Localized increase of chemokines in the lumen of human cerebral aneurysms. Stroke 2013;44:2594-2597.

27 García-Rodríguez LA, Gaist D, Morton J, Cookson C, González-Pérez A: Antithrombotic drugs and risk of hemorrhagic stroke in the general population. Neurology 2013;81: 566-574. gra A, Dorhout Mees SM, et al: Randomized controlled trial of acetylsalicylic acid in aneurysmal subarachnoid hemorrhage: the MASH study. Stroke 2006;37:2326-2330.

-29 Chalouhi N, Jabbour P, Magnotta V, Hasan D: The emerging role of ferumoxytol-enhanced MRI in the management of cerebrovascular lesions. Molecules 2013;18:96709683.

30 Hasan DM, Mahaney KB, Magnotta VA, et al: Macrophage imaging within human cerebral aneurysms wall using ferumoxytol-enhanced MRI: a pilot study. Arterioscler Thromb Vasc Biol 2012;32:1032-1038.

31 Casado-Arroyo R, Sostres C, Lanas A: Optimizing the use of aspirin for cardiovascular prevention. Drugs 2013;73:803-814.

-32 Moncada S, Higgs EA, Vane JR: Human arterial and venous tissues generate prostacyclin (prostaglandin $\mathrm{x}$ ), a potent inhibitor of platelet aggregation. Lancet 1977;1:18-20.

33 Patrono C, Coller B, FitzGerald GA, Hirsh J, Roth G: Platelet-active drugs: the relationships among dose, effectiveness, and side effects: the seventh ACCP conference on antithrombotic and thrombolytic therapy. Chest 2004;126(3 suppl):234S-264S

-34 Ricciotti E, FitzGerald GA: Prostaglandins and inflammation. Arterioscler Thromb Vasc Biol 2011;31:986-1000.

35 McAdam BF, Mardini IA, Habib A, et al: Effect of regulated expression of human cyclooxygenase isoforms on eicosanoid and isoeicosanoid production in inflammation. J Clin Invest 2000; 105:1473-1482.

- 36 Smyth EM, Grosser T, Wang M, Yu Y, FitzGerald GA: Prostanoids in health and disease. J Lipid Res 2009;50(suppl):S423-S428.

37 Langenbach R, Loftin C, Lee C, Tiano H: Cyclooxygenase knockout mice: models for elucidating isoform-specific functions. Biochem Pharmacol 1999;58:1237-1246.

38 Morteau O, Morham SG, Sellon R, et al: Impaired mucosal defense to acute colonic injury in mice lacking cyclooxygenase-1 or cyclooxygenase-2. J Clin Invest 2000;105:469478.

39 Mulay SR, Gaikwad AB, Tikoo K: Combination of aspirin with telmisartan suppresses the augmented TGFbeta/smad signaling during the development of streptozotocin-induced type I diabetic nephropathy. Chem Biol Interact 2010;185:137-142.

40 Lapponi MJ, Carestia A, Landoni VI, et al: Regulation of neutrophil extracellular trap formation by anti-inflammatory drugs. J Pharmacol Exp Ther 2013;345:430-437.

41 Kaiser J: Will an aspirin a day keep cancer away? Science 2012;337:1471-1473.

42 Berg M, Søreide K: Prevention: will an aspirin a day keep the colorectal cancer away? Nat Rev Clin Oncol 2011;8:130-131

43 Jacobs EJ: Will an aspirin a day help keep fatal cancer away? Lancet 2011;377:3-4. 
44 Garcia-Albeniz X, Chan AT: Aspirin for the prevention of colorectal cancer. Best Pract Res Clin Gastroenterol 2011;25:461-472.

45 Thun MJ, Namboodiri MM, Heath CW Jr: Aspirin use and reduced risk of fatal colon cancer. N Engl J Med 1991;325:1593-1596.

-46 Antithrombotic Trialists' Collaboration: Collaborative meta-analysis of randomised trials of antiplatelet therapy for prevention of death, myocardial infarction, and stroke in high risk patients. BMJ 2002;324:71-86.

-47 Beckman JA, Creager MA, Libby P: Diabetes and atherosclerosis: epidemiology, pathophysiology, and management. JAMA 2002; 287:2570-2581.

-48 Freestone T, Turner RJ, Coady A, Higman DJ, Greenhalgh RM, Powell JT: Inflammation and matrix metalloproteinases in the enlarging abdominal aortic aneurysm. Arterioscler Thromb Vasc Biol 1995;15:1145-1151.

49 Starke RM, Chalouhi N, Ali MS, et al: The role of oxidative stress in cerebral aneurysm formation and rupture. Curr Neurovasc Res 2013; 10:247-255.

50 Chimowitz MI, Lynn MJ, Derdeyn CP, et al: Stenting versus aggressive medical therapy for intracranial arterial stenosis. N Engl J Med 2011;365:993-1003.
51 Wong KS, Chen C, Fu J, et al: Clopidogrel plus aspirin versus aspirin alone for reducing embolisation in patients with acute symptomatic cerebral or carotid artery stenosis (CLAIR study): a randomised, open-label, blindedendpoint trial. Lancet Neurol 2010;9:489497.

52 Grines CL, Bonow RO, Casey DE Jr, et al: Prevention of premature discontinuation of dual antiplatelet therapy in patients with coronary artery stents: a science advisory from the American Heart Association, American College of Cardiology, Society for Cardiovascular Angiography and Interventions, American College of Surgeons, and American Dental Association, with representation from the American College of Physicians. Circulation 2007;115:813-818.

53 Kikano GE, Brown MT: Antiplatelet therapy for atherothrombotic disease: an update for the primary care physician. Mayo Clin Proc 2007;82:583-593.

54 Wang Y, Wang Y, Zhao X, Liu L, et al: Clopidogrel with aspirin in acute minor stroke or transient ischemic attack. N Engl J Med 2013; 369:11-19.

55 Chimowitz MI, Lynn MJ, Howlett-Smith H, et al: Comparison of warfarin and aspirin for symptomatic intracranial arterial stenosis. N Engl J Med 2005;352:1305-1316.
56 Hankey GJ, Norman PE, Eikelboom JW: Medical treatment of peripheral arterial disease. JAMA 2006;295:547-553.

57 Hiatt WR, Krantz MJ: The efficacy of aspirin in peripheral arterial disease: an unresolved question. J Mal Vasc 2007;32:71-72.

58 Berger JS, Krantz MJ, Kittelson JM, Hiatt WR: Aspirin for the prevention of cardiovascular events in patients with peripheral artery disease: a meta-analysis of randomized trials. JAMA 2009;301:1909-1919.

59 Poredos P, Jezovnik MK: Is aspirin still the drug of choice for management of patients with peripheral arterial disease? Vasa 2013;42: 88-95.

60 Ait-Oufella H, Wang Y, Herbin O, et al: Natural regulatory $\mathrm{T}$ cells limit angiotensin II-induced aneurysm formation and rupture in mice. Arterioscler Thromb Vasc Biol 2013;33: 2374-2379.

61 Vorp DA, Lee PC, Wang DH, et al: Association of intraluminal thrombus in abdominal aortic aneurysm with local hypoxia and wall weakening. J Vasc Surg 2001;34:291-299.

62 Lindholt JS, Björck M, Michel JB: Anti-platelet treatment of middle-sized abdominal aortic aneurysms. Curr Vasc Pharmacol 2013;11: 305-313. 